\title{
Promoting Natural Interactions Through Embedded Input Using Novel Sensing Techniques
}

\author{
Sang Ho Yoon \\ School of Mechanical Engineering, Purdue University \\ West Lafayette, IN 47907 \\ yoon87@purdue.edu
}

\begin{abstract}
From mobile devices to interactive objects, various input methods are provided using built-in motion and capacitive touch sensors. These inputs are offered in effective and efficient manner where users can operate interface quickly and easily. However, they do not fully explore the input space supported by human's natural motion behavior. As a solution, my work focuses on promoting natural interaction through hand-driven embedded input powered by multimodal and magnetic sensing techniques. In my previous works, embedded inputs were implemented in the form of smart textile, stylus, and ring supporting from mobile devices to everyday objects. Throughout the paper, I will briefly go over implemented systems along with evaluated results and potential applications. Future research direction is highlighted at the end of the paper.
\end{abstract}

\section{Author Keywords}

Input Device; Wearables; Embedded Interaction; Sensing

Technique; Mobile Interaction; Magnetism

\section{ACM Classification Keywords}

H.5.2. Information Interfaces and Presentation (e.g. HCI): User Interfaces

\section{INTRODUCTION}

Recent development in computing power, display, and sensors have resulted in providing enhanced interface in terms of mobility and performance. Researchers also utilize the affordance and the availability of everyday objects to implement user interfaces. To support upcoming trends in user interfaces with various form factors, previous studies suggested on utilizing natural correspondences like human motion behaviors [7]. To incorporate natural human motion behaviors as an input, recent works explore new input metaphors such as eyes-free [1], around device [3], and object interactions [6]. Still, there are rooms to improve performance, richness, and affordance using novel sensing techniques.

Permission to make digital or hard copies of part or all of this work for personal or classroom use is granted without fee provided that copies are not made or distributed for profit or commercial advantage and that copies bear this notice and the full citation on the first page. Copyrights for third-party components of this work must be honored. For all other uses, contact the Owner/Author. Copyright is held by the owner/author(s). UIST'16 Adjunct, October 16-19, 2016, Tokyo, Japan

ACM 978-1-4503-4531-6/16/10.

http://dx.doi.org/10.1145/2984751.2984782
Mark Wesier [13] mentioned that the best technology is "weave themselves into the fabric of everyday life". To this extent, my dissertation focuses on enhancing the performance, richness, and affordance of interactions using embedded inputs. As a first step, I came up with a multimodal sensing smart textile to support an eyes-free input based on somatosensory tactility of the finger. The proposed prototype showed performance improvement in terms of response times and an accuracy while achieving high satisfaction from users. Secondly, the magnetic sensing has been explored deeply to offer a full 3D position tracking around the mobile device using a stylus. By enabling 3D volume around the mobile device interactive, we support upcoming interfaces like mobile augmented reality (AR). Recent work focuses on making plain objects interactive by simply embedding a small magnet. With a finger-worn device, instant and customizable user interface can be implemented with any given objects. The simple setup allows users to easily implement interface with everyday objects while preserving rich interactions.

\section{RELATED WORKS}

\section{Interaction Through Finger-Worn Devices}

Within the hand, the fingers have been explored widely as an interaction medium since it represents human intents precisely with high flexibility. Previously, a finger-worn controller is implemented with tactile buttons, capacitive touch sensors, and bend sensors [12]. Although this work supports rich interactions through a single finger, it would be difficult to maintain a comfort due to attached physical components. Other sensing technique like computer vision approach has been widely explored. However, a line-of-sight view of hands or markers should be maintained within the optical sensing volume. In our approach [15], we suggest a finger operating wearable device which utilizes somatosensory tactility based on a passive haptic feedback to enhance eyes-free mobile interactions.

\section{Position Tracking with Magnetic Sensing Technique}

Magnetic sensing has been explored extensively for position tracking using both active and passive magnetic sources. Although active magnetic sources provide accurate position tracking, they require a stationary hardware setup which limits user's mobility. On the other hand, passive magnetic sources have provided enhanced mobile inputs without requiring an extensive hardware setup $[4,8]$. However, recent approaches still require either extra hardware modifications on the mobile device or desktop-level computing power. 
These requirements limit scalability and practicability as a stand-alone input. In TMotion [14], we developed a stylus embedded with an inertial measurement unit (IMU) and a permanent magnet to fully support 3D position tracking around the mobile device without any hardware modifications.

\section{Everyday Object Interaction}

Performing interactions using everyday objects offers task affordances while maintaining current working environment. This creates an opportunity for instant and casual interactions where naturally embedded behavior with existing object transforms to a meaningful input method [9]. To this extent, $i$ Con utilized fixed cameras to use everyday objects as an auxiliary controller and instant interface [5]. However, previous approaches require a room-level or object-level hardware setup that limits the availability and the acceptance of interactions. In our recent work, we utilize a magnetic sensing technique to bring an interactivity to existing objects instantly with high flexibility in customization.

\section{RESEARCH GOALS}

\section{Hand-Driven Embedded Input}

Hand has been a dominant input medium for interacting with digital devices: 1) mobile devices adopt a finger touch for general input, 2) new interface including AR and VR focus on bringing a natural hand motion as input, and 3) input accessories like styli and joysticks are operated by hand. To this extent, I have focused on developing embedded inputs operable by a hand. To preserve the comfort of using hand, the new sensing techniques require seamless interaction that does not negatively affect the natural hand usage. To this end, my previous works provide embedded inputs considering prototype wearability, coherence with existing input methods, and minimal instrumentation requirements.

\section{Augmenting Interaction with Sensing Technique}

Recent gaming applications starts to utilize hands and whole body gestures based on a 3D depth camera. However, the overall mobile devices and interactive objects are still limited to the flat screen or the surface due to a lack of depth camera. In addition, a computer vision approach requires a line-ofsight view which is hard to maintain during interacting with mobile devices or objects. For this reason, I explore the magnetic tracking which provides an around-device interaction without requiring a line-of-sight view. On the other hand, the richness in interactions using a human finger is limited. To fulfill the richness of interface, I focus on novel sensing techniques using finger wearables to obtain rich inputs while preserving a simple form factor.

\section{Instant and Customizable Interface}

Often new sensing techniques require a new set of hardware installation on the device or the user. This limits the sustainability and flexibility of employing new approaches. Moreover, customizing interface conventionally requires physical modifications on the prototype including a hardware components rearrangement or a new assembly. In my research, I aim on employing new sensing techniques while minimizing the needs of physical hardware installations. With suggested

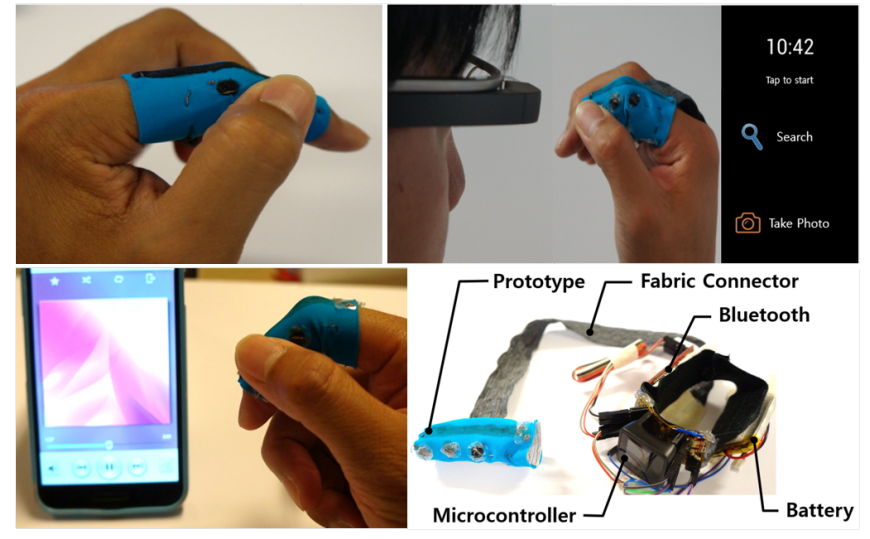

Figure 1. A finger-worn smart textile prototype provides a multimodal sensing (finger bending and pressure) to support an eyes-free interaction during daily activities such as exercising, driving, and working

solution, new sensing techniques are more readily acceptable to existing devices as well as users.

\section{SYSTEM IMPLEMENTATION}

To fulfill above research goals, I have implemented three different embedded input system. These input metaphors are either directly or indirectly related to the use of human hands. In this section, my research works are explained in detail.

\section{Finger-worn Textile Input Device}

In my earliest work, I implement a multimodal sensing fingerworn input using a single layer of the textile [15]. Utilizing strain and pressure characteristics of the carbon elastomer, two types of finger motions are defined for interactions including finger pressing and bending. To support these input signals, the prototype adopts a two-phase and a polynomial regression analysis. These algorithms are used to model the relationship between magnitudes of pressure and strain against applied finger pressing and bending. With a multilevel threshold, the prototype recognizes different levels of pressure and strain sensing. Simple gestures like a swipe are captured via the algorithm based on the temporal position tracking. By using the prototype with a two-finger interaction, total of 14 or more distinct input signals are created. To preserve better tactility and wearability comparing to existing data gloves equipped with hard and rigid components, we

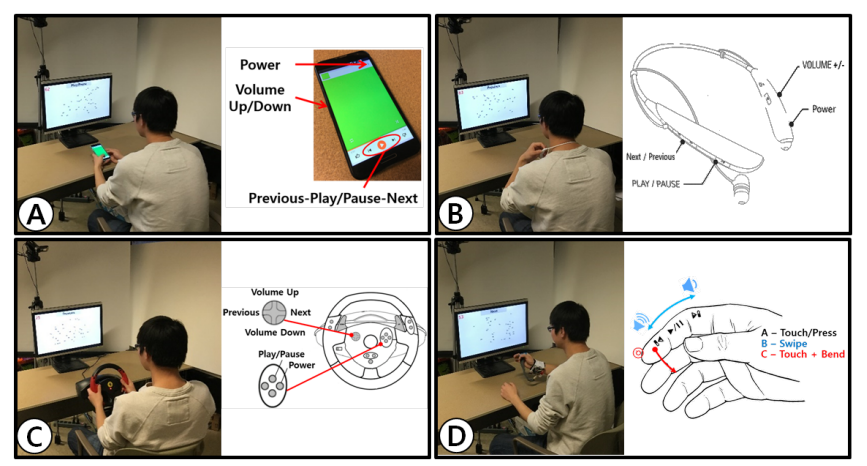

Figure 2. Multitasking user study is carried out to compare performance with existing input devices 


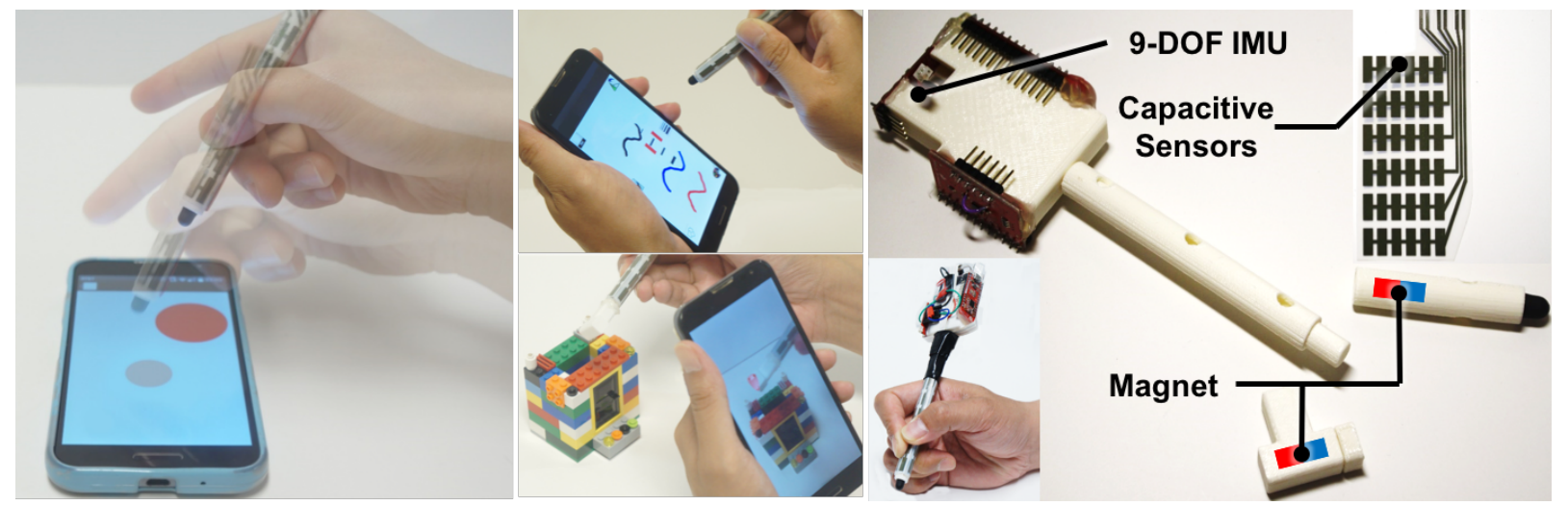

Figure 3. With magnetic sensing technique, we enable a real-time 3D position tracking using an embedded magnet and an IMU with an unmodified mobile device. Tmotion enlarges interaction spaces above and behind the device and provides rich interactions with 3D position tracking. The prototype is implemented in a form of stylus.

utilize elastic and soft textile. The use of elastic textile entitles the robustness of performance while providing proper fixation onto the finger.

To explore the user performance of proposed input in terms of a response time and an accuracy, a multitasking user study is carried out with existing inputs such as phone, Bluetooth headset, and steering wheel. User study is carried out under visually distracted conditions. The study results indicate that the proposed input is less susceptible to the visual distraction comparing to conventional input devices. Since the proposed input encourages use of proprioception from fingers, users can maintain operation performance. This work shows how the multimodal sensing can be utilized in augmenting sensing capability and input performance using a single finger.

\section{Embedded 3D Input using Magnetic Sensing}

In the subsequent work, magnet sensing technique is explored to enable 3D position tracking around the mobile device. A prototype in a form of stylus is embedded with a magnet and an IMU. The magnetic field vector and the orientation of the embedded magnet are used to compute the magnet's position relative to the mobile device. Lastly, a 3D position tracking with rate greater than $30 \mathrm{~Hz}$ is achieved only using a single magnetometer from the mobile device side. In interactionwise, the prototype supports continuous/discontinuous interactions in above/behind the device spaces.

The experiment shows an average error of $4.55 \mathrm{~mm}$ in the space of $80 \mathrm{~mm} \times 120 \mathrm{~mm} \times 100 \mathrm{~mm}$. The tracking robustness is also confirmed under the dynamic tracings and various orientations. The interaction performances by users are verified with tracking precision and targeting accuracy. The potential applications such as the around-device interaction using 3D volumes around the mobile device and input control in mobile AR are implemented. Throughout this work, interactions are augmented by utilizing a 3D input space and tracking natural hand motions with the stylus.

\section{Instant and Customizable Finger-worn Input}

In my recent work, a finger-worn device based on magnetic sensing is implemented to provide instant and customizable

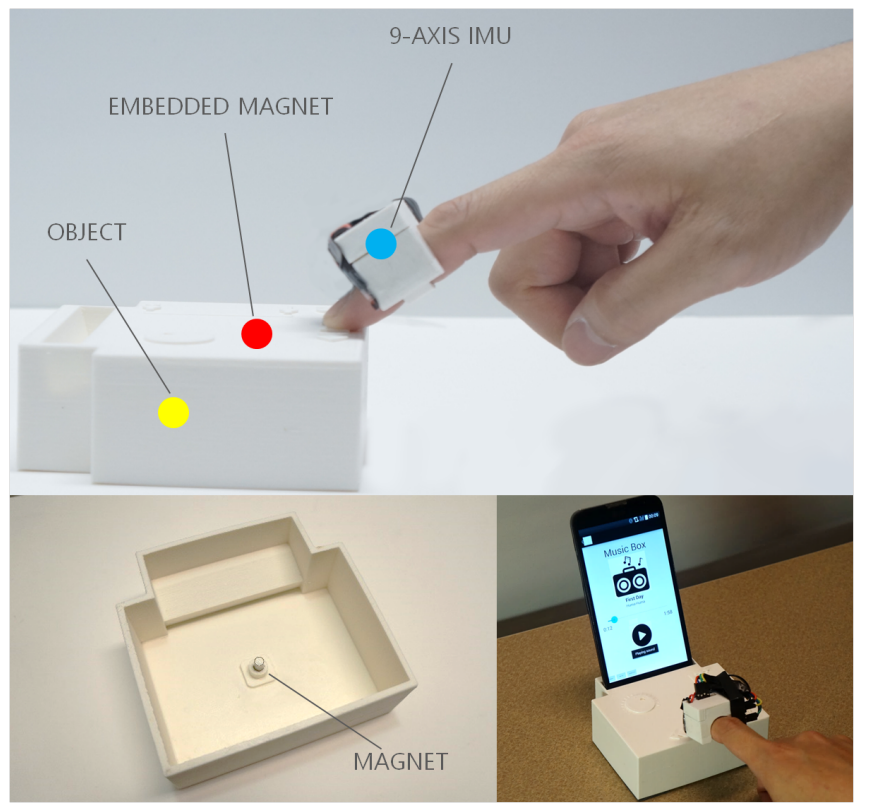

Figure 4. Recent work demonstrates a finger-worn device with magnetic sensing technique to track fingertip around a permanent magnet. By embedding a magnet to the object, we bring interactivity to objects where we utilize the affordances existing objects.

interface with plain objects. The prototype supports both fabricated and everyday objects as an interaction medium. Along with the position tracking based on the magnetic sensing, a particle filter is employed to track the finger tip near the permanent magnet. This allows us to simply inserting or attaching a small magnet to bring interactivity to the target object. The discrete and the continuous input types are offered through a finger tip based on position tracking capability.

The software tool is also provided to compute the optimal magnet location in a 3D model with a given interface set. Using this approach, users do not need to modify hardware setup where only magnet location can be altered upon interface change. The evaluation confirms high-precision tracking performance while user study validates the input performance in general control tasks. This work can benefit novice inter- 
action designers and general users who want to implement interface instantly with flexibility in customization.

\section{POTENTIAL RESEARCH DIRECTION}

With a forthcoming evolution in sensor performance and machine learning algorithm, new sensing techniques can be explored. To further support the research claim on "Promoting natural interactions through hand-driven embedded inputs", I plan to investigate following research topics in the future.

\section{Bio-signal Sensing}

Different forms of bio-signals such as electromyography (EMG), pressure distribution around body parts, and skin deformation can be thoroughly extracted with recent sensor development. Previous approaches $[10,11]$ suggest the use of bio-signals for the hand-driven embedded input. These works exploit natural human motions based on bio-signal processing for human machine interface. Inspired from these works, I will focus on hand related bio-signal sensing to provide rich and robust inputs while preserving affordances.

\section{Machine Learning Integration}

Machine learning approaches [2] have been utilized to classify user intents with the data from large set of sensors. This approach has shown effectiveness to classify a set of small number of input commands. With the aid of emerging machine learning methods like support vector machine (SVM) and deep convolution neural network (DCNN), richer input commands based on human's natural motion behavior can be recognized. To this end, I will explore capability of machine learning to integrate with various sensing techniques.

\section{CONCLUSION}

My dissertation has presented how hand-driven embedded inputs improve performance, richness, and affordance of interactions. Novel sensing techniques such as multimodal (finger bending and pressure) and magnetic sensing have been exploited to augment the inputs. To preserve the comfort and the acceptability of provided input methods, I focus on embedding prototypes to hand related input metaphors in forms of smart textile, stylus, and ring. The evaluations and user studies have confirmed the superior input performance of each project and potential applications are illustrated using proposed inputs.

I envision that upcoming interfaces like AR and VR can be benefited from augmented interaction through hand-driven embedded input. With the development of sensor technology, various sensing techniques can be utilized to improve the existing input metaphors.

\section{ACKNOWLEDGMENTS}

I am grateful for the guidance of my advisor Prof. Karthik Ramani. I would also thank all of my collaborators. This work was supported by the National Science Foundation (NSF) IGERT Grant \#1144843. Any opinions, findings, and conclusions or recommendations expressed in this material are those of the authors and do not necessarily reflect the views or opinions of the funding agency.

\section{REFERENCES}

1. Brewster, S., Lumsden, J., Bell, M., Hall, M., and Tasker, S. Multimodal' eyes-free'interaction techniques for wearable devices. In Proc. of CHI'03, ACM (2003), 473-480.

2. Burges, C. J. A tutorial on support vector machines for pattern recognition. Data mining and knowledge discovery 2, 2 (1998), 121-167.

3. Butler, A., Izadi, S., and Hodges, S. Sidesight: multi-touch interaction around small devices. In Prof. of UIST'08, ACM (2008), 201-204.

4. Chen, K.-Y., Lyons, K., White, S., and Patel, S. utrack: $3 \mathrm{~d}$ input using two magnetic sensors. In Proc. of UIST'13, ACM (2013), 237-244.

5. Cheng, K.-Y., Liang, R.-H., Chen, B.-Y., Laing, R.-H., and Kuo, S.-Y. icon: utilizing everyday objects as additional, auxiliary and instant tabletop controllers. In Prof. of CHI'10, ACM (2010), 1155-1164.

6. Fitzmaurice, G. W., Ishii, H., and Buxton, W. A. Bricks: laying the foundations for graspable user interfaces. In Proc. of CHI'95, ACM Press/Addison-Wesley Publishing Co. (1995), 442-449.

7. Hand, C. A survey of $3 d$ interaction techniques. In Computer graphics forum, vol. 16, Wiley Online Library (1997), 269-281.

8. Liang, R.-H., Cheng, K.-Y., Su, C.-H., Weng, C.-T., Chen, B.-Y., and Yang, D.-N. Gausssense: attachable stylus sensing using magnetic sensor grid. In Proc. of UIST'12, ACM (2012), 319-326.

9. Pohl, H., and Rohs, M. Around-device devices: my coffee mug is a volume dial. In Proc. of MobileHCI'14, 81-90.

10. Rekimoto, J. Gesturewrist and gesturepad: Unobtrusive wearable interaction devices. In Proc. of ISWC'01, IEEE (2001), 21-27.

11. Saponas, T. S., Tan, D. S., Morris, D., Balakrishnan, R., Turner, J., and Landay, J. A. Enabling always-available input with muscle-computer interfaces. In Proc. of UIST'09, ACM (2009), 167-176.

12. Tsukadaa, K., and Yasumurab, M. Ubi-finger: Gesture input device for mobile use. Ubicomp 2001 Informal Companion Proceedings (2001), 11.

13. Weiser, M. The computer for the 21 st century. Scientific american 265, 3 (1991), 94-104.

14. Yoon, S. H., Huo, K., and Ramani, K. Tmotion: Embedded 3d mobile input using magnetic sensing technique. In Proc. of TEI'16, ACM (2016), 21-29.

15. Yoon, S. H., Huo, K., and Ramani, K. Wearable textile input device with multimodal sensing for eyes-free mobile interaction during daily activities. Pervasive and Mobile Computing (2016). 